\title{
Article \\ Solution of fractional autonomous ordinary differential equations
}

\author{
Rami AlAhmad ${ }^{1, t, \neq * \mathbb{O}, \text { Qusai AlAhmad }}{ }^{2, \ddagger}$ and Ahmad Abdelhadi ${ }^{3, \ddagger}$ \\ 1 Mathematics department, Yarmouk University, Irbid, Jordan; rami_thenat@yu.edu.jo \\ 2 Mathematics Department, California state university at Northridge, Northridge, CA 91330-8313, USA; \\ qussai.alahmad.781@my.csun.edu \\ * Correspondence: rami_thenat@yu.edu.jo \\ + Current address: Department of Mathematics and natural sciences, Higher colleges of technology, Ras \\ AlKhaimah, UAE \\ $\ddagger$ These authors contributed equally to this work.
}

Abstract: Autonomous differential equations of fractional order and non-singular kernel are solved. While solutions can be obtained through numerical, graphical, or analytical solutions, we seek an implicit analytical solution.

Keywords: Fractional derivatives, Caputo fractional derivative, the Caputo-Fabrizio fractional derivative, Laplace transform

MSC: 44A10,26A33

\section{Introduction}

Fractional calculus has resurfaced and gained momentum due to its potential in engineering systems, multidisciplinary fields, biology, medicine, and applied sciences. Its wide range of applications includes areas like linear anomalous diffusion equation and its characteristics [1], modeling biological phenomena, respiratory tissue, and drug diffusion [2], and recently the global impact of the corona virus (COVID-19)and epidemic models [3]. At the same time, fractional calculus has found its way to sensors, analog filters and digital filters [4].

The autonomous ordinary differential equations

$$
\frac{d y}{d t}=F(y(t))
$$

play a major role in Engineering, Physics, Biology, and other fields like economics and medicine. The solution to (1.1), for the given initial condition $y\left(t_{0}\right)=y_{0}$, is

$$
y(t)=y\left(t_{0}\right)+\int_{t_{0}}^{t} \frac{d \xi}{F(y(\xi))} .
$$

The logistic differential equation $y^{\prime}(t)=y(t)(1-y(t))$ represents a special case of (1.1). It's an autonomous ordinary differential equation with a wide range of engineering applications.With an initial condition $y(0)=1 / 2$, the logistic differential equation yields the solution

$$
y(t)=\frac{1}{1+e^{-t}} .
$$

Recent advancements in calculus have allowed for different presentations of the autonomous equation (1.1). In particular, the fractional representations of the autonomous differential equation, , see [5-7]. Contrary to the Riemann-Liouville fractional deriva- 
tive, the Cupato fractional derivative is more suitable to applied problems. The initial conditions are properly defined. The Caputo fractional derivative is defined as

$$
\left({ }^{C} D^{\alpha} f\right)(t)=\frac{1}{\Gamma(n-\alpha)} \int_{0}^{t} \frac{f^{(n)}(\tau) d \tau}{(t-\tau)^{\alpha+1-n}}
$$

where $n-1<\alpha \leq n$.

In this paper, we proceed to study the following fractional version of the autonomous differential equation (1.1):

$$
\left({ }^{C F} D^{\alpha} y\right)(t)=F(y(t))
$$

where ${ }^{C F} D^{\alpha}$ is the Caputo-Fabrizio fractional derivative. We provide an example of a differential fractional version of the exponential growth function. Our findings are an extension to the results found in [7].

\section{Preliminaries}

M. Caputo and M. Fabrizio presented a definition of fractional derivative with a non singular kernel:

For a real smooth function $f$ and for $\alpha \in[0,1]$, the Caputo-Fabrizio fractional derivative [9] is given by

$$
\left({ }^{C F} D^{\alpha} f\right)(t)= \begin{cases}\frac{1}{1-\alpha} \int_{0}^{t} e^{-\frac{\alpha}{1-\alpha}(t-u)} f^{\prime}(u) d u, & 0 \leq \alpha<1 \\ f^{\prime}(t), & \alpha=1\end{cases}
$$

According to this definition, the following are the Caputo-Fabrizio fractional derivatives for some elementary functions.

Proposition 2.1. For $f(t)=c$, then $\left({ }^{C F} D^{\alpha} f\right)(t)=0$.

Proposition 2.2. For $f(t)=t$, then $\left({ }^{C F} D^{\alpha} f\right)(t)=\frac{1-e^{-\frac{\alpha t}{1-\alpha}}}{\alpha}$.

Proposition 2.3. For $f(t)=t^{r} ; \Re(r)>-1$, then

$$
\left({ }^{C F} D^{\alpha} f\right)(t)=\frac{1}{1-a} e^{-\frac{a t}{1-a}}\left(\frac{a}{1-a}\right)^{-r-1} \gamma\left(r+1, \frac{a t}{1-a}\right) .
$$

Proposition 2.4. For $f(t)=e^{b t}$, then $\left({ }^{C F} D^{\alpha} f\right)(t)=\frac{b\left(e^{\frac{a t}{a-1}}-e^{b t}\right)}{a(b-1)-b}$.

The following proposition gives the Laplace transform of the Caputo-Fabrizio fractional derivative. The following proposition defines the "antiderivative" of the Caputo-Fabrizio fractional derivative.

Proposition 2.5. Let ${ }^{C F} D^{\alpha}$ be the Caputo-Fabrizio fractional derivative. Let $g(t)=\left({ }^{C F} D^{\alpha} f\right)(t)$, then

$$
f(t)=f(0)+(1-\alpha) g(t)+\int_{0}^{t} g(\xi) d \xi .
$$

Proof. Since $g(t)=\left({ }^{C F} D^{\alpha} f\right)(t)$, then

$$
g(t)=\frac{1}{1-\alpha} \int_{0}^{t} e^{-\frac{\alpha}{1-\alpha}(t-u)} f^{\prime}(u) d u
$$

Therefore,

$$
\int_{0}^{t} e^{\frac{\alpha u}{1-\alpha}} f^{\prime}(u) d u=(1-\alpha) e^{\frac{\alpha t}{1-\alpha}} g(t) .
$$


Differentiate both sides to get that,

$$
f^{\prime}(t)=(1-\alpha) g^{\prime}(t)+\alpha g(t)
$$

Integrating both sides and using $g(0)=0$ give the desired result.

Proposition 2.6. Let ${ }^{C F} D^{\alpha}$ be the Caputo-Fabrizio fractional derivative. The Laplace transform of $\left({ }^{C F} D^{\alpha} f\right)(t)$ is $\frac{1}{\alpha+s(1-\alpha)}(s F(s)-f(0)) ; \Re(s)>\frac{\alpha}{\alpha-1}$, where $F(s)$ is the Laplace transform of $f(t)$.

Proof. Using (2.1), $g(t)=\left({ }^{C F} D^{\alpha} f\right)(t)$ satisfies

$$
(1-\alpha) e^{\frac{\alpha t}{1-\alpha}} g(t)=\int_{0}^{t} e^{\frac{\alpha u}{1-\alpha}} f^{\prime}(u) d u .
$$

Differentiating both sides of (2.2) and simplify to get:

$$
(1-\alpha) g^{\prime}(t)+\alpha g(t)=f^{\prime}(t)
$$

Taking Laplace transform for both sides implies:

$$
(1-\alpha) s G(s)+\alpha G(s)=s F(s)-f(0),
$$

where $F(s)=L_{t}[f(t)](s)$ and $G(s)=L_{t}[g(t)](s)$.

Now, solving (3.2) for $G(s)$, the result follows.

This result is read as

$$
\left({ }^{C F} D^{\alpha} f\right)(t)=L^{-1}\left[\frac{1}{\alpha+s(1-\alpha)}(s F(s)-f(0))\right],
$$

where $L^{-1}$ is the inverse Laplace transform.

Example 2.7. In this example, we solve a fractional version of the exponential growth (decay) differential equation $y^{\prime}=k y$. Consider the differential equation

$$
\left({ }^{C F} D^{\alpha} y\right)(t)=k y(t)
$$

To solve this equation, take the Laplace transform for both sides of this equation to get:

$$
\frac{1}{\alpha+s(1-\alpha)}(s Y(s)-y(0))=k Y(s) \text {. }
$$

Solving this equation for $Y(s)$ implies that

$$
Y(s)=\frac{y(0)}{((\alpha-1) k+1) s-\alpha k} .
$$

Therefore, the solution of $(2.5)$ is $y(t)=C e^{\frac{\alpha k t}{1-(1-\alpha) k}}$.

\section{The solution of the autonomous fractional differential equation}

Theorem 3.1. The solution of the autonomous fractional differential equation

$$
\left({ }^{C F} D^{\alpha} y\right)(t)=F(y(t))
$$


is given implicitly as

$$
\int_{y\left(t_{0}\right)}^{y(t)} \frac{d u}{F(u)}-(1-\alpha) \ln \left(\frac{F(y(t))}{F\left(y\left(t_{0}\right)\right)}\right)=\alpha\left(t-t_{0}\right) .
$$

Proof. Let $y(t)$ be a solution of (3.1). Then the definition (2.1) gives:

$$
\frac{1}{1-\alpha} e^{-\frac{\alpha}{1-\alpha} t} \int_{0}^{t} e^{\frac{\alpha}{1-\alpha} u} y^{\prime}(u) d u=F(y(t)) .
$$

Equivalently,

$$
\int_{0}^{t} e^{\frac{\alpha}{1-\alpha} u} y^{\prime}(u) d u=(1-\alpha) e^{\frac{\alpha}{1-\alpha} t} F(y(t)) .
$$

Differentiate both sides and simplify to get:

$$
y^{\prime}(t)=(1-\alpha) F^{\prime}(y(t)) y^{\prime}(t)+\alpha F(y(t)) .
$$

Rearrange the terms as:

$$
\frac{y^{\prime}(t)}{F(y(t))}-(1-\alpha) \frac{F^{\prime}\left(y(t) y^{\prime}(t)\right)}{F(y(t))}=\alpha .
$$

Integrate both sides from $t_{0}$ to $t$ to get:

$$
\int_{t_{0}}^{t} \frac{y^{\prime}(u)}{F(y(u))} d u-(1-\alpha) \int_{t_{0}}^{t} \frac{F^{\prime}\left(y(u) y^{\prime}(u)\right)}{F(y(u))} d u=\alpha \int_{t_{0}}^{t} 1 d u .
$$

Use integration by substitution for the integrals in the left side to get the desired result.

Example 3.2. To solve (2.5), use (3.2) to get that

$$
\int_{y\left(t_{0}\right)}^{y(t)} \frac{d u}{k u}-(1-\alpha) \ln \left(\frac{k y(t)}{k y\left(t_{0}\right)}\right)=\alpha\left(t-t_{0}\right) .
$$

Therefore,

$$
\ln (y(t))-k(1-\alpha) \ln y(t)=k \alpha t+C
$$

Hence,

$$
(1-k(1-\alpha)) \ln (y(t))=k \alpha t+C .
$$

This implies the solution of (2.5) is

$$
y(t)=C e^{\frac{k \alpha t}{1-k(1-\alpha)}} .
$$

It yields same result as in Example 2.7.

Example 3.3. We construct the solution of the fractional logistic differential equation and plot for different values of $\alpha$

$$
\left({ }^{C F} D^{\alpha} y\right)(t)=y(t)(1-y(t)) .
$$

According to (3.2), the solution is implicitely given as

$$
\int_{y\left(t_{0}\right)}^{y(t)} \frac{d u}{u(1-u)}-(1-\alpha) \ln \left(\frac{y(t)(1-y(t))}{y\left(t_{0}\right)\left(1-y\left(t_{0}\right)\right)}\right)=\alpha\left(t-t_{0}\right) .
$$


After simplifications, we will get:

$$
y^{\alpha}(1-y)^{\alpha-2}=C e^{\alpha t}
$$

where $C$ is a constant which depends on $\alpha$ and the initial conditions $t_{0}$ and $y\left(t_{0}\right)$. If we assume that $y(0)=1 / 2$ then the resluting solution is

$$
y^{\alpha}(1-y)^{\alpha-2}=4^{1-\alpha} e^{\alpha t} .
$$

The set of solutions, for different values of $\alpha$, along with the solution of the logistic differnetial equation (1.2) are represented by the figure 1.

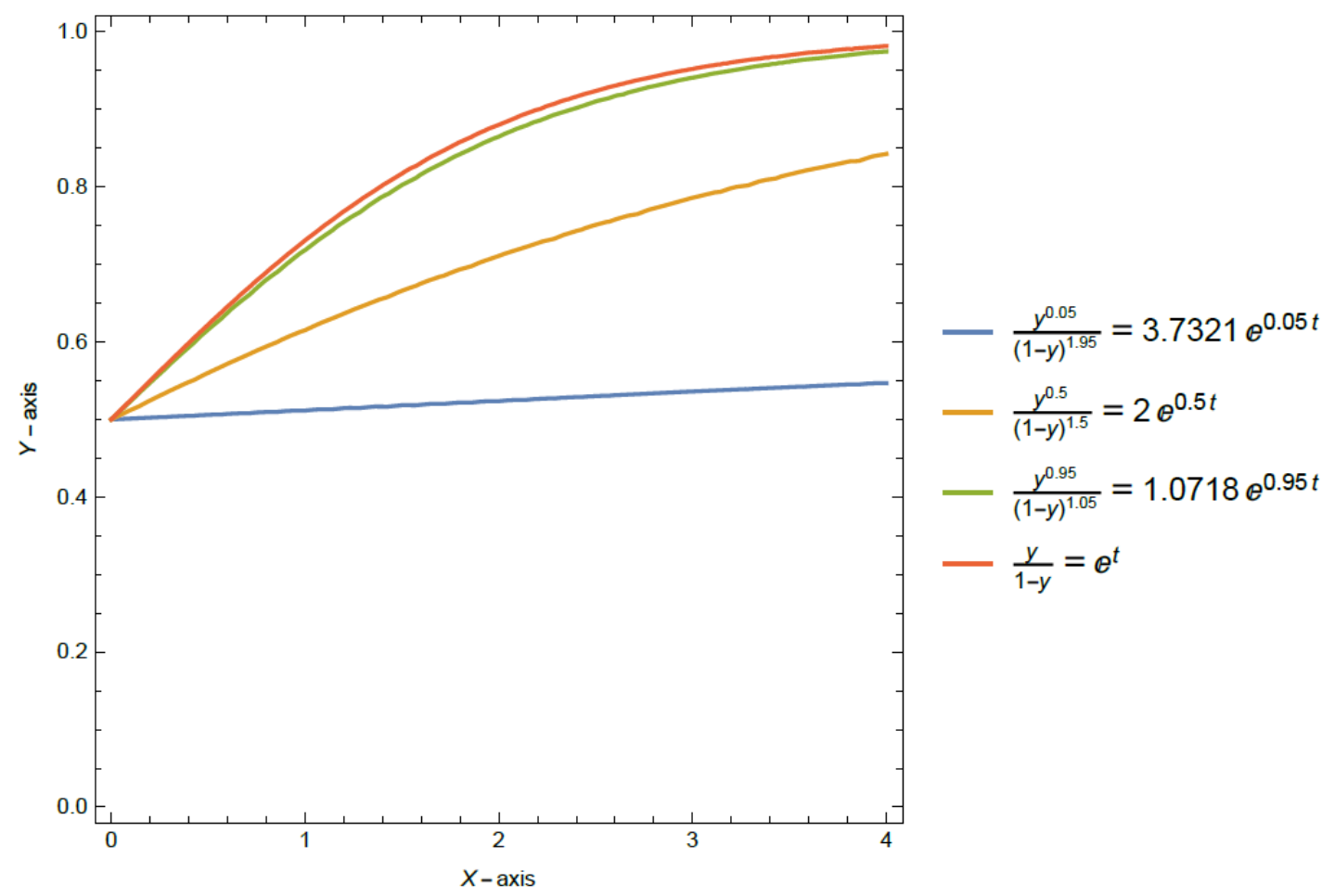

Figure 1. solutions of logistic fractional differential equation (3.8) with $\alpha=0.05,0.5,0.95,1$ in [8].

The same solution of the logistic fractional differential equation was obtained previously

Example 3.4. We construct a solution to the fractional autonomous differential equation

$$
\left({ }^{C F} D^{\alpha} y\right)(t)=1+y^{2}(t)
$$

According to (3.2), the solution is implicitly given as

$$
\int_{y\left(t_{0}\right)}^{y(t)} \frac{d u}{1+u^{2}}-(1-\alpha) \ln \left(\frac{1+y^{2}(t)}{\left.1+y^{2}\left(t_{0}\right)\right)}\right)=\alpha\left(t-t_{0}\right) .
$$

After simplifications, we will get:

$$
\arctan (y)-(1-\alpha) \ln \left(1+y^{2}\right)=\alpha t+C .
$$

If we assume that $y(0)=0$ then the resulting solution is

$$
\arctan (y)-(1-\alpha) \ln \left(1+y^{2}\right)=\alpha t
$$


The set of solutions, for different values of $\alpha$, along with the solution of the differential equation $y^{\prime}=1+y^{2}$ are represented by the figure 2 .

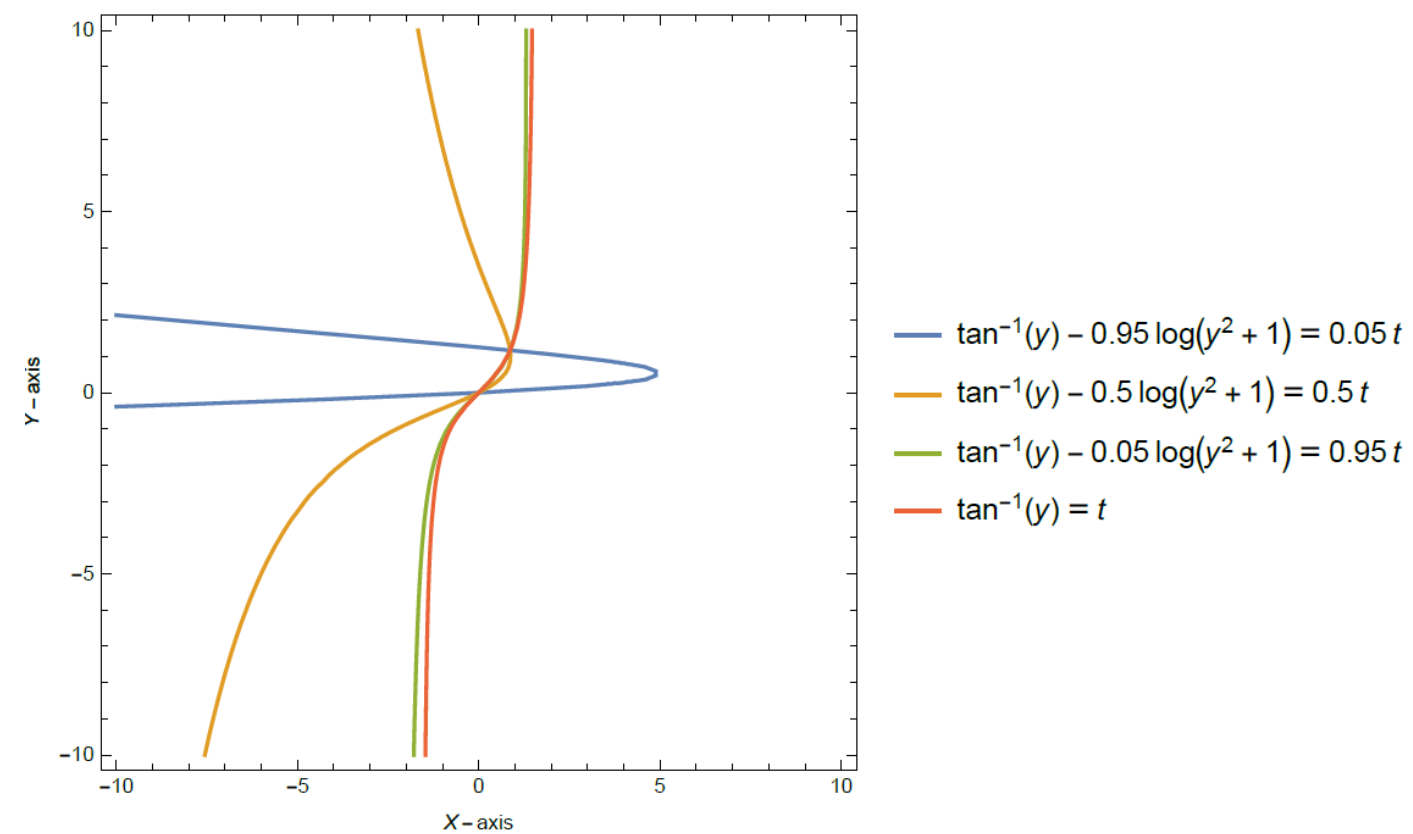

Figure 2. solutions of the autonomous fractional differential equation (3.10) with $\alpha=$ $0.05,0.5,0.95,1$

\section{Conclusion}

Using the Caputo-Fabrizio fractional derivative we have shown a solution can be obtained for different examples of the fractional autonomous differential equations. For given initial conditions, we presented sets of solutions for different values of between 0 and 1 . We have arrived at the same solution as [8].

Author Contributions: R.AlAhmad; methodology,Q. AlAhmad; software, R.AlAhmad; formal analysis, Q.AlAhmad; investigation, A.Abdelhadi; data curation, A.Abdelhadi; writing-review and editing, R.AlAhmad.; supervision

Funding: This research received no external funding

Conflicts of Interest: The authors declare no conflict of interest.

\section{References}

1. R. K. Gazizov,S. Y. Lukashchuk, Higher-order symmetries of a time-fractional anomalous diffusion equation. Mathematics 2021, 9, 3. doi:10.3390/ math9030216.

2. C. Ionescu, A. Lopes, D. Copot, J. A. T. Machado, J. H. T. Bates, The role of fractional calculus in modeling biological phenomena: A review. Communications in Nonlinear Science and Numerical Simulations 2017, 51, 141-159. doi:10.1016/j.cnsns.2017.04.001.

3. Y. Chen, F. Liu, Q. Yu, T. Li, Review of fractional epidemic models, Applied Mathematical Modelling 2021, 97, 281-307. https:/ /doi.org/10.1016/j.apm.2021.03.044.

4. C. I. Muresan, I. R. Birs, E. H. Dulf, D. Copot, L. Miclea, A review of recent advances in fractional-order sensing and filtering techniques, Sensors 2021, 21, 281-307. doi:10.3390/s21175920.

5. Brauer, F., Castillo-Chavez, C., Feng, Z. Mathematical models in epidemiology. Mathematical models in epidemiology, 2019.

https:/ / public.ebookcentral.proquest.com/choice/publicfullrecord.aspx?p=5941284.

6. H. R. Thieme, Mathematics in Population Biology, Princeton University Press, 2018. doi:10.1515/9780691187655. 
7. J. T. Machado, V. Kiryakova, F. Mainardi, Recent history of fractional calculus,Communications in Nonlinear Science and Numerical Simulation2011, 16, 1140-1153. doi:https:// doi.org/10.1016/j.cnsns.2010.05.027.

8. J. J. Nieto, Solution of a fractional logistic ordinary differential equation, Applied Mathematics Letters, 2022, 123. doi:https: / / doi.org/10.1016/j.aml.2021.107568.

9. M. Caputo, M. Fabrizio, On the singular kernels for fractional derivatives. some applications to partial differential equations, Progress in Fractional Differentiation and Applications, 2021, 7, 79-82. doi:10.18576/PFDA/070201. 
